\title{
Microfabricated Torsional Actuators Using Self-Aligned Plastic Deformation of Silicon
}

\author{
Jongbaeg Kim, Hyuck Choo, Liwei Lin, Member, IEEE, Fellow, ASME, and \\ Richard S. Muller, Life Fellow, IEEE, Member, ASME
}

\begin{abstract}
In this paper, we describe angular vertical-combdrive torsional microactuators made in a new process that induces residual plastic deformation of single-crystal-silicon torsion bars. Critical dimensions of the vertically interdigitated moving-and fixed-comb actuators are self-aligned in the fabrication process and processed devices operate stably over a range of actuation voltages. We demonstrate MEMS scanning mirrors that resonate at $2.95 \mathrm{kHz}$ and achieve optical scan angles up to 19.2 degrees with driving voltages of $40 \mathrm{~V}_{\mathrm{dc}}$ plus $13 \mathrm{~V}_{\mathrm{pp}}$. After continuous testing of five billion cycles at the maximum scanning angle, we do not observe any signs of degradation in the plastically deformed silicon torsion bars.

[1550]
\end{abstract}

Index Terms-Electrostatic actuators, plastic deformation, selfalignment, scanning micromirrors, torsional actuators, vertically interdigitated combs.

\section{INTRODUCTION}

$\mathbf{R}$ ESEARCHERS have used electrostatic comb-drive actuators for many diverse MEMS applications. Measured performance of these actuators has proven their capabilities for achieving extended ranges-of-movement stably and reliably over a wide range of resonant frequencies, and they have been especially useful for optical scanners and switches. For example, lateral comb-drives with mechanical hinges or linkages made of polycrystalline- or single-crystalline silicon have been used to drive torsional actuators [1], [2]. Experiments with these devices revealed reliability problems and limitations on maximum operational frequencies. In one design for torsional actuators Hah and co-workers interleaved vertically aligned comb-finger sets with planarized polysilicon structures [3] using a process employing chemical-mechanical polishing (CMP) to achieve both higher frequencies and larger scan angles than those obtained in earlier designs built with lateral comb-drives. Other vertically aligned comb-drive designs have been fabricated using polysilicon-on-SOI [4] or single-crystal silicon coupled with a wafer-bonding technique [5]. Krisnamurthy and Solgaard demonstrated a self-aligned vertical comb [6] made using wafer-bonding, grinding, and polishing, in addition to an anisotropic-etch step of single-crystal silicon.

Manuscript received March 18, 2005; revised November 10, 2005. This paper was presented in part at the 12th International Conference on Solid-State Sensors, Actuators and Microsystems (Transducers '03, Boston, MA, June 9-12, 2003). Subject Editor H. Zappe.

J. Kim is with the School of Mechanical Engineering, Yonsei University, Seoul, Korea (e-mail: jongbaeg@gmail.com).

H. Choo, L. Lin, and R. S. Muller are with Berkeley Sensor and Actuator Center, University of California, Berkeley, CA 94720 USA (e-mail: r.muller@ieee.org).

Digital Object Identifier 10.1109/JMEMS.2006.876789
Collectively, the vertical-comb-drive actuators described above can be described as staggered vertical comb-drive (SVC) actuators [5]. SVCs commonly face a severe fabrication challenge posed by their need for precise alignment between the two comb-finger layers.

Recent research has demonstrated that when the material forming the two vertical combs is not co-planar, but rather angled relative to one another, the achievable torsional displacement is significantly increased. When the comb geometries are similar, for example, these angular vertical comb-drive (AVC) actuators have been driven with maximum $50 \%$ more torsional displacement than the nonangled SVC types [7], [8]. The fabrication processes for AVCs start by defining stationary and movable comb structures in the same silicon layer. Next, either the stationary comb structure or the movable comb structure is deflected into an offset position. The deflection process has, in previous work, been induced either through residual stress by adding a metal overlayer [7] or through the surface-tension forces that result from reflowing a patterned-photoresist layer [8]. For the metal overlayer method to be effective, the structure to be deformed must be sufficiently flexible for the applied force to induce a useful offset value. This requirement directly affects the resonant frequencies of the deformed structures, limiting them in practice to a few hundreds of Hz. In addition, for the polymer hinges, precisely controlling the reflow of the hinge material and consistently achieving good mechanical reliability have proven to be the challenging tasks. More recently, AVCs were fabricated using intentionally induced stiction on the designated plates giving quite limited offset angle between stator and rotor combs [9], [10]. These methods are simple to get AVC, but the achievable initial tilt angle is quite limited since they are using limited thickness of buried oxide layer (typically, $1-2 \mu \mathrm{m}$ ) for the stiction plates to be displaced for angular tilting of the mirror.

Our research demonstrates a new way to build AVC torsional actuators that show higher resonant frequencies as well as other performance advantages. We use single-crystal silicon and achieve the angled orientation between comb fingers by deforming one of the pair plastically in an anneal step [11]. MEMS researchers have, in recent years, demonstrated several applications for plastic deformation in silicon including the fabrication of domes [12] and deformed membranes [13] in polycrystalline and single-crystal silicon under the pressure of heated gases. Plastically deformed polysilicon structures have also been used in a self-assembled MEMS process [14]. On a larger scale, silicon-chip-mounting using plastically deformed single-crystal-silicon cantilever beams has been 


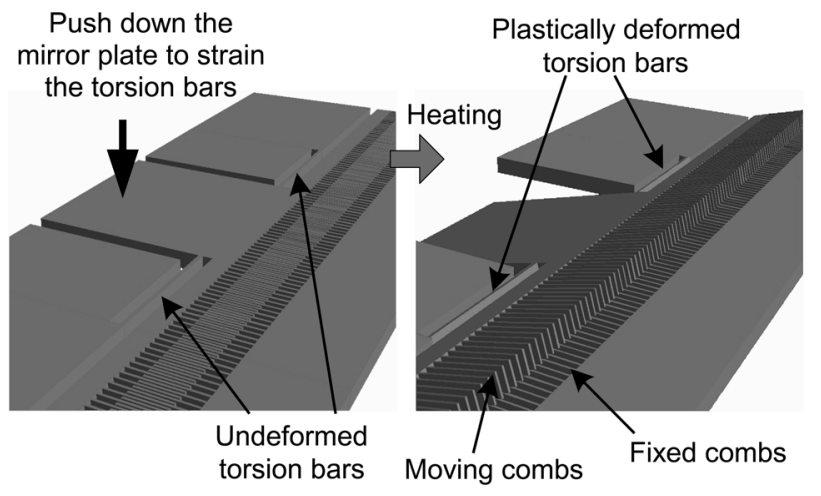

Fig. 1. Batch-processed plastic deformation of microactuator torsion bars (before and after plastic deformation of torsion bars).

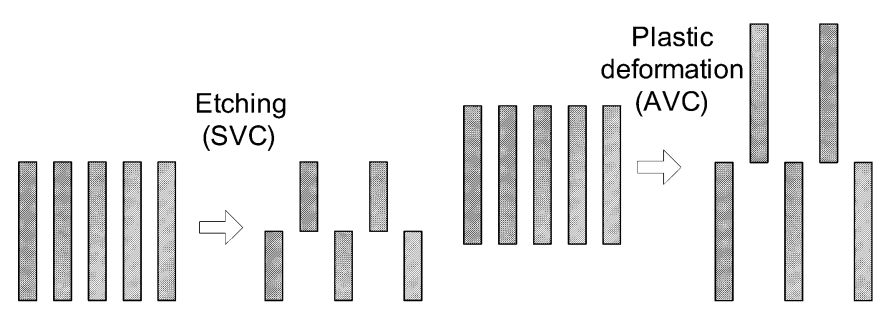

(a)

(b)

Fig. 2. Higher aspect ratio of vertical comb fingers by new fabrication approach; taller comb fingers (and larger actuation angle) are achieved with plastic deformation process than with conventional SVC fabrication.

demonstrated [15]. In our paper, self-aligned vertically interdigitated comb-sets are fabricated in a precisely controlled, repeatable batch process.

We describe both the fabrication process and the dynamic performance of the actuators, using them to drive scanning micromirrors. The scanners have resonant frequencies that are an order-of-magnitude higher than those reported in earlier AVCs based upon residual stress process. Measurements on scanning mirrors made with these AVCs have confirmed their repeatable and stable operation.

\section{DESIGN AND FABRICATION}

A torsional scanning-mirror actuator is composed of four components to generate mirror motions out of the substrate plane; the mirror plate, the torsion bars, the moving comb set, and the fixed comb set. An ideal scanning-mirror actuator should have smooth and flat mirror surface for optical quality and precisely aligned moving- and fixed-comb sets for stable operation. Our design for AVC scanning mirrors provides both of these requirements. Fig. 1 shows schematically how, in our process, the torsion bars are permanently deformed and actuated. Initially, both the moving- and fixed-combs, formed in the same device layer of an SOI wafer, are coplanar. In the coplanar configuration, voltages applied between the comb fingers cannot cause vertical displacement, and therefore in the following step, the combs attached to mirror structures are depressed from their original orientations by pushing on them with a separately prepared silicon substrate. This substrate is processed separately so that it has a precisely spaced array of pillar structures. The pillars are spaced such that each of them

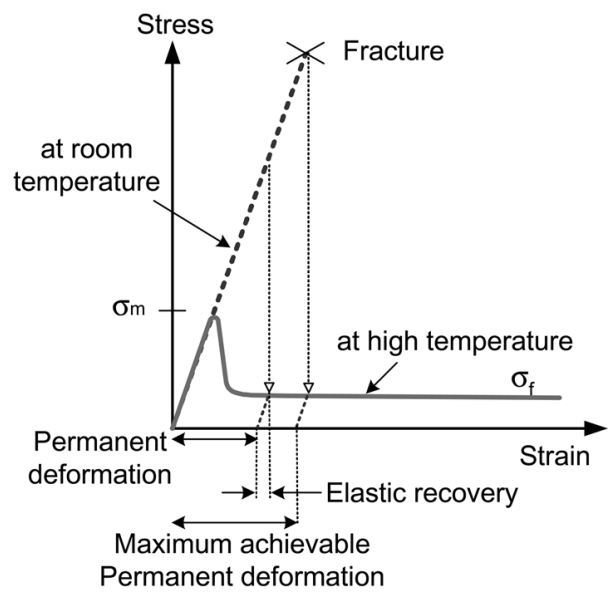

Fig. 3. The permanent plastic deformation and elastic recovery explained in a stress-strain curve of silicon where $\sigma_{m}$ is maximum yield stress, $\sigma_{f}$ is flow stress, the stress needed to continue plastic deformation, at an elevated temperature. (From [11].)
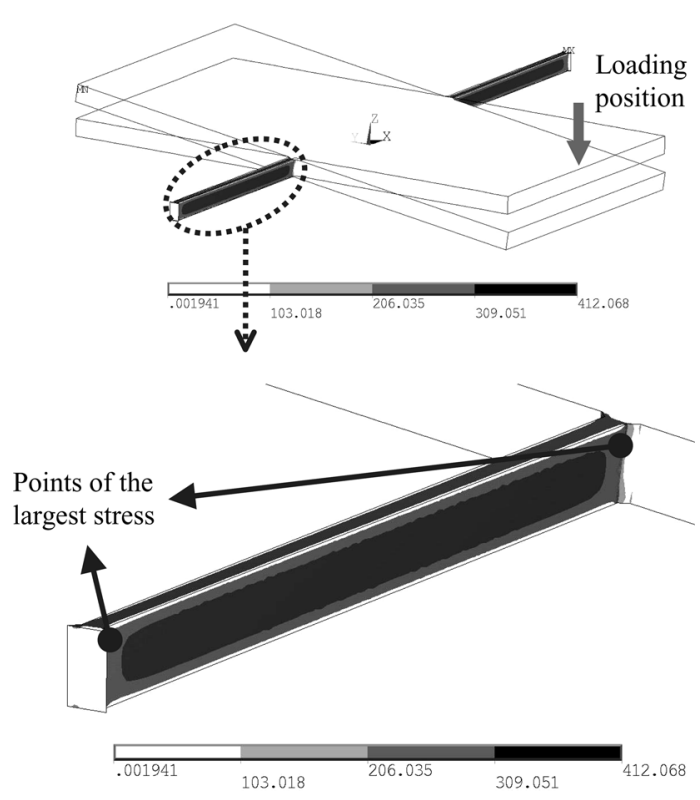

Fig. 4. Stress distribution in the torsion bars; stress induced in the mirror is negligibly small- the maximum stress on the mirror is less than $10 \mathrm{Mpa}$ except the boundary between the mirror and torsion bars (unit of the scale bar: Mpa).

deforms one pair of torsion bars by pushing the associated mirror edge downward from its original position (parallel to its comb partner). A subsequent high-temperature $\left(900{ }^{\circ} \mathrm{C}\right.$ ) annealing step converts the initially elastic deformations of the torsion bars that connect the two comb finger sets into permanent plastic deformations [Fig. 1(b)]. With the comb pairs in this angled configuration, voltages applied between them will induce torque in the torsion-bar supports that connect them.

Our new AVC, shown in Fig. 1, has several advantages over previous SVC actuators. Fig. 2(a) and (b) shows typical fabrication processes for SVCs and AVCs, respectively, to produce self-aligned comb fingers. In these structures, a high aspect ratio for the comb fingers is desired to produce a large actuation range, and the achievable aspect ratio is essentially limited to that which can be obtained using deep-reactive-ion-etching (DRIE). In our fabrication process, we can displace one comb 


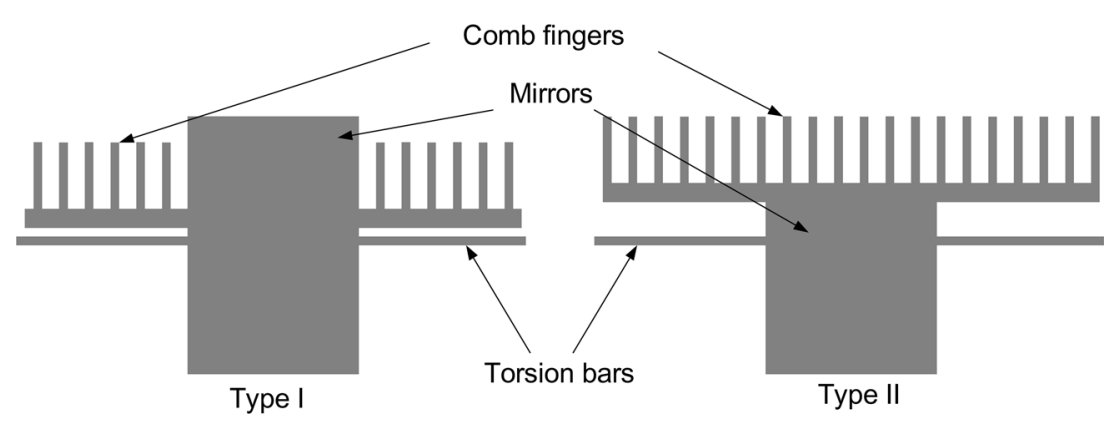

Fig. 5. Two base designs of scanning mirror actuators.

TABLE I

Typical Design Examples of Two Types of AVCs BuILt AND Studied In THIS ResEARCH

\begin{tabular}{|l|l|l|}
\hline Unit: $\mu \mathrm{m}$ & Type I & Type II \\
\hline Device Thickness & $50 \mu \mathrm{m}$ & $50 \mu \mathrm{m}$ \\
\hline Mirror Size $(\mathrm{WxL})$ & $800 \mu \mathrm{m} \times 1500 \mu \mathrm{m}$ & $1000 \mu \mathrm{m} \times 1000 \mu \mathrm{m}$ \\
\hline Torsional Spring $(\mathrm{WxL})$ & $16 \mu \mathrm{m} \times 800 \mu \mathrm{m}$ & $16 \mu \mathrm{m} \times 750 \mu \mathrm{m}$ \\
\hline Comb Finger Dimension $(\mathrm{WxL})$ & $8 \mu \mathrm{m} \times 240 \mu \mathrm{m}$ (overlap & $10 \mu \mathrm{m} \times 300 \mu \mathrm{m}$ (overlap \\
& length: $160 \mu \mathrm{m})$ & 1 length: $200 \mu \mathrm{m})$ \\
\hline Number of Rotor Comb Fingers & 70 & 120 \\
\hline Gap between Comb Fingers & $8 \mu \mathrm{m}$ & $10 \mu \mathrm{m}$ \\
\hline
\end{tabular}

finger set through its entire thickness and thereby achieve the comb finger aspect ratio twice as large as those in SVCs. Therefore, the range of actuation can be also larger under the similar conditions such as torsion bar dimension, location of comb fingers and operation voltage.

Fig. 3 shows a typical stress-strain curve measured in singlecrystal silicon at high temperature [13]. At room temperature, silicon is a brittle material with a yield stress of GPa order. At elevated temperatures, its mechanical properties change; for example, its maximum yield stress $\left(\sigma_{\mathrm{m}}\right)$ decreases due to the increased mobility of dislocations in the crystal. At temperature exceeding roughly $600{ }^{\circ} \mathrm{C}$, silicon structures begin to deform plastically and the flow stress $\sigma_{\mathrm{f}}$ (the stress needed to continue plastic deformation) decreases with rising temperature. For our AVC-process, we have designed the pillar structures such that the torsion bars are stressed above $\sigma_{\mathrm{m}}$ at the annealing temperature, causing them to yield plastically. Fig. 4 shows the room-temperature stress distribution in the torsion bar supporting a mirror when the mirror comb-finger is angled at $4^{\circ}$ to its mate. The distribution is calculated using finite-element analysis on the torsion bars that measure $16 \mu \mathrm{m} \times 50 \mu \mathrm{m} \times 600$ $\mu \mathrm{m}$ and support a mirror of $800 \mu \mathrm{m} \times 1400 \mu \mathrm{m}$. Fixed boundary condition was set at the ends of torsion bars that are connected to anchors and a point load is applied at the edge of the mirror to simulate the real case loading where a mirror is pushed down by a pillar. The deformation process also induces stresses in the mirror plate; however, their magnitudes are much smaller than $\sigma_{\mathrm{m}}$ or $\sigma_{\mathrm{f}}$ and negligible compared to the stresses in the torsion bars. The maximum stress in the mirror plate was calculated to be smaller than $10 \mathrm{Mpa}$ even at the positions close to the torsion bars, while the stresses in the torsion bars are well above 150-200 MPa. During the high-temperature anneal, the stresses in the torsion bars are relieved by plastic deformation, but the mirror plates are unaffected.

Two scanning-mirror designs that we fabricated and studied are shown in Fig. 5 where they are designated as type I and II.
For type I AVCs, a torsion bar connects the mirror to comb fingers at the middle of the mirrors while in type II, the torsion bars are off-centered from the mirror and all the comb fingers are on the other side of the torsion bar to balance the mass moment of inertia with respect to the axis of rotation. The bigger size and symmetry with respect to the rotation axis of the mirror in type I design allows slight misalignment of incident light on the mirror while the resonant frequency is lower due to the larger mass moment of inertia. In type II, more comb fingers were involved for larger actuation forces and faster scanning is expected compared to type I. Table I provides design data for the type I and II devices.

Fig. 6 shows all steps in our AVC fabrication process. We start the fabrication process by defining the moving- and fixed-comb fingers in the same device layer of an SOI substrate [Fig. 6(a)-(c)]. During the DRIE step, not only the comb-drive actuators, but also the key-slots, shown in Fig. 6(b) are formed. A backside-etching step carried out underneath the mirrors and combs removes sufficient silicon to provide clearance for the mirror motion. Alignment for this backside-etch step is not critical; misalignments are tolerable as long as the backside etch area is designed to provide enough clearance for the mirror motion. In the next processing step, we make use of a second chip processed by single step of DRIE that has been configured with pillars to displace the comb structures to the desired tilt angles. On this chip are protrusions (keys) that align to key-slots in the comb-actuator chip when the two are brought together, sandwich style, and placed into a furnace. The temperature of the furnace is linearly increased by $15{ }^{\circ} \mathrm{C} / \mathrm{min}$ rate up to $900^{\circ} \mathrm{C}$, and this temperature is maintained for $30 \mathrm{~min}$ inducing plastic deformation of the torque-loaded torsion bars. The furnace is then cooled down to room temperature in $1 \mathrm{~h}$. The whole annealing process is performed at nitrogen environment to prevent unnecessary oxidation of the silicon surface. After separation from the processing substrate, the comb pairs are angled one to another as shown in Fig. 6(f). No critical align- 


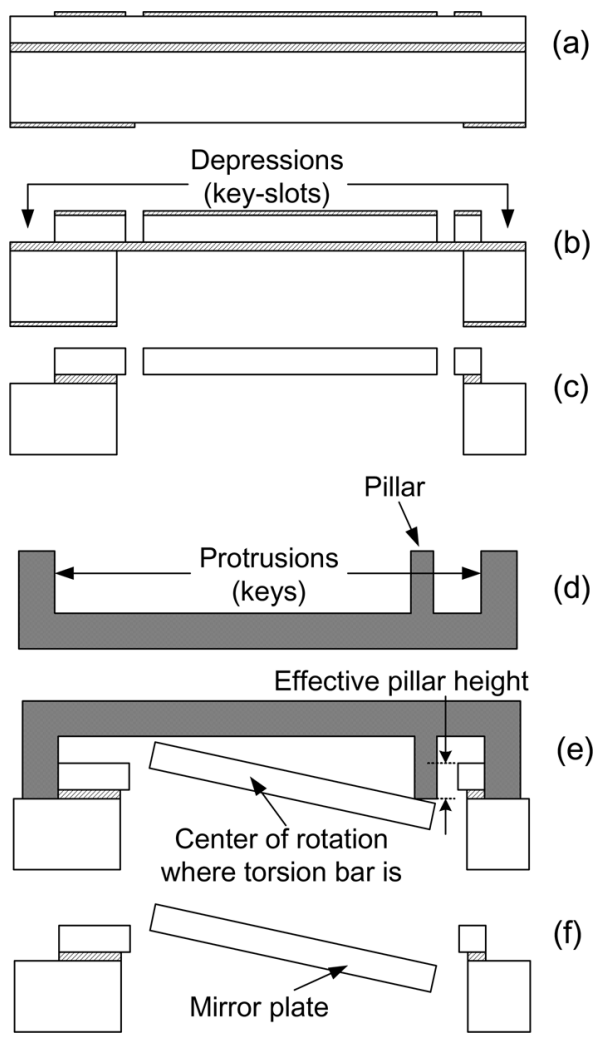

Fig. 6. Process flow for plastically deformed vertical actuators. (a) Pattern thermal-oxide layers on top and bottom surfaces of SOI wafer. (b) Etch device and handle layers of SOI wafer using DRIE. (c) Remover thermal-oxide layers and exposed buried oxide layer. (d) Create pillar structures on the lid wafer using DRIE. (e) Place lid wafer on the device wafer and heat the stacked wafers to $900^{\circ}$ in furnace. (f) Cool down and remove the lid wafer.

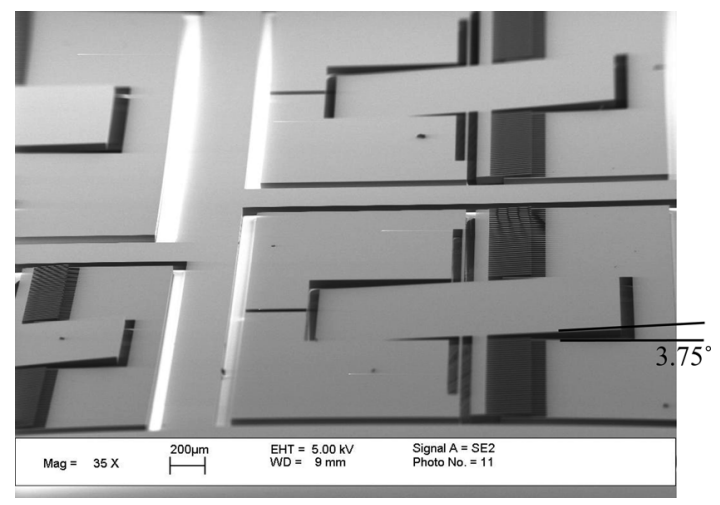

Fig. 7. SEM picture of AVC with scanning mirrors made using new process.

ment steps are necessary to carry out the process that we have described. The comb-finger pairs are self-aligned because they are defined in a single masking step, and the device and lid chips are precisely assembled by the lithographically defined keys and key-slots. No damage in the pillars or processing chips is observed indicating that these processing chips could be used repeatedly.

Fig. 7 is an SEM microphotograph of batch-fabricated micromirrors produced by our plastic-deformation process. A close-up view of tilted comb fingers is presented in Fig. 8, showing the precisely aligned vertical-comb sets. Fig. 9 is a close-up view of a plastically deformed torsion bar showing

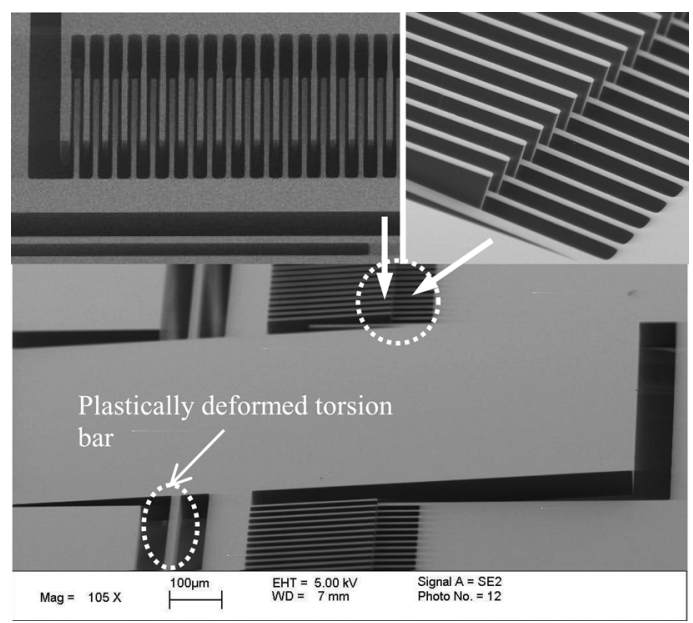

Fig. 8. Plastically tilted mirror and precisely aligned vertical combs. The upper-left photo shows the top view of comb fingers and the upper-right photo is the close-up oblique view of the angular vertical combs.

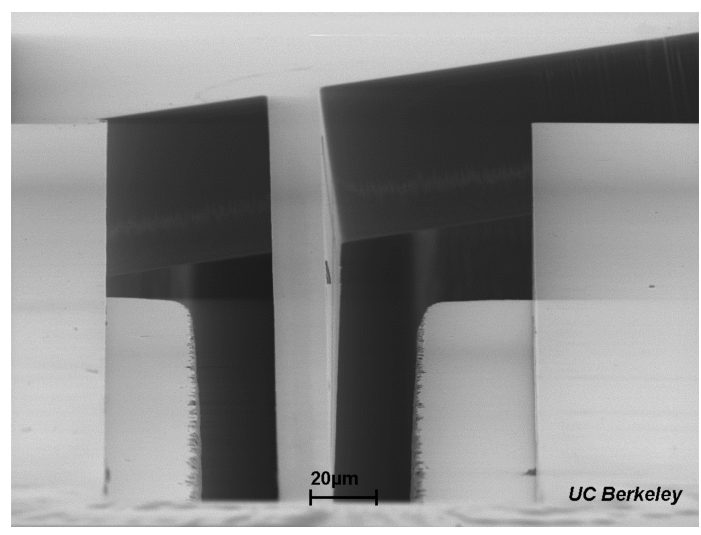

Fig. 9. Close-up view of plastically deformed torsion bar.

that the deformation appears to be uniform along the torsion bar. We did not observe cracking in any of the torsion bars. Fig. 10 shows pillars formed on the processing chip used to deform the microactuators in Fig. 7.

The maximum initial tilt angle that can be formed by the method of plastic deformation that we have described above is limited by the fracture strength of the single-crystal silicon at room temperature. For example, the torsion bar dimensions presented in Table I easily allows $20-30^{\circ}$ of elastic deformation without fracture, and more than $90 \%$ of the angles can be turned into plastically deformed initial tilt angle. These elastically deformed angles are determined both by the height of the pillars on the processing chip and by the locations at which the pillars push on the mirror (Fig. 11). Clearly, a given tilt angle can be induced either by using a taller pillar and applying the force at point $\mathrm{P}$ or by using a shorter pillar and applying the force at point $\mathrm{Q}$. Although the tilt angles are the same in these two cases (as, therefore, are the torque values in the torsion bar), the forces (and, hence, the stresses) in the torsion bars are not equal.

For the two cases described earlier, the corresponding forces $F_{P}$ and $F_{Q}$ vary inversely with their moment arm distances $F_{P} / F_{Q}=x_{Q} / x_{P}$. These vertical forces are balanced by equal forces in the torsion bar causing it to deflect downward (as a doubly supported beam loaded at its midpoint) which leads to 


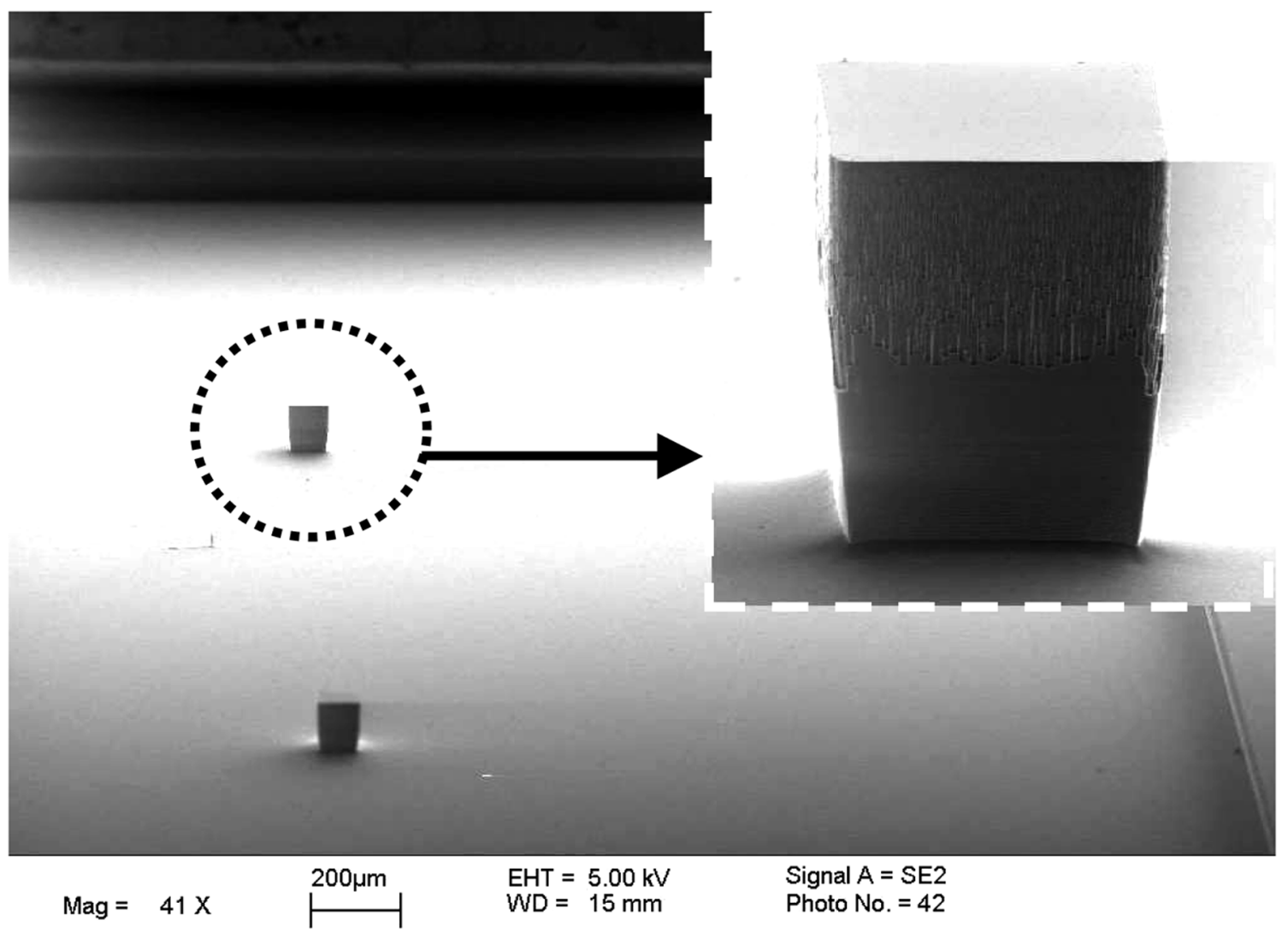

Fig. 10. Micropillar structures fabricated on the lid wafer; the right side inset shows the close-up of a pillar.

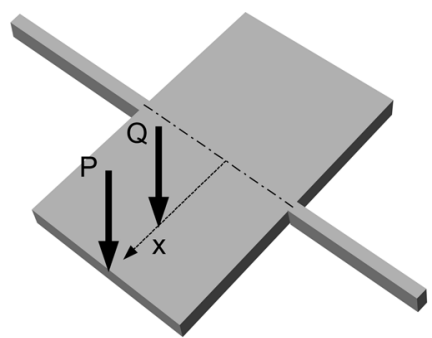

(a)

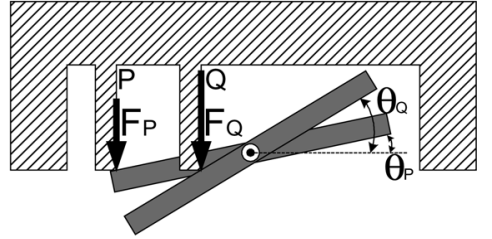

(b)

Fig. 11. Different forces applied on the mirror surface depending on the position and height of the pillar structure.

unwanted bending of the torsion bar or distortion in mirror surfaces. To reduce this undesirable effect, the pillars should load the mirrors at the longest moment arm or, therefore, at the mirror tips. In our type II design with $5.22^{\circ}$ initial-angle deflection, the measured vertical deflections of torsion bars after plastic deformation using $50 \mu \mathrm{m}$ pillars were smaller than $0.3 \mu \mathrm{m}$.

Fig. 12(a) presents the three-dimensional (3-D) image generated by white light interferometric measurement (WYKO NT3300). The measured radii-of-curvature of the mirrors using WYKO were in the order of meters, which indicate there can be only very little deformation or warpage induced in the mirror plate after high temperature plastic deformation process. Another concern is the probable scratches on the mirror surfaces when the pillar structures push down the mirrors. It was impossible to point out the points-of-contact on the mirror exactly since there were no observable scratches or damages found under WYKO or SEM inspection. The surface roughness of the mirror was also measured on the presumptive contact positions of the pillar using an atomic force microscope (AFM) as shown in Fig. 12(b). The average roughness was less than $4 \mathrm{~nm}$ for the measured positions, which is a typical value for polished SOI wafer surface.

\section{DEVICE CHARACTERIZATION AND DISCUSSION}

As depicted in Fig. 3, silicon has a finite modulus of elasticity even at an elevated temperature at which the yield strength is reduced. This means that plastic deformation does not completely relax the strain in the torsion bar and, therefore, there is some elastic recovery upon removal of the load after cooldown. This "springback" results in the mirror initial angular deflections being smaller than the values set by the pillars on the processing chips. We investigated springback by fabricating a series of identically sized mirrors having different torsion-bar 


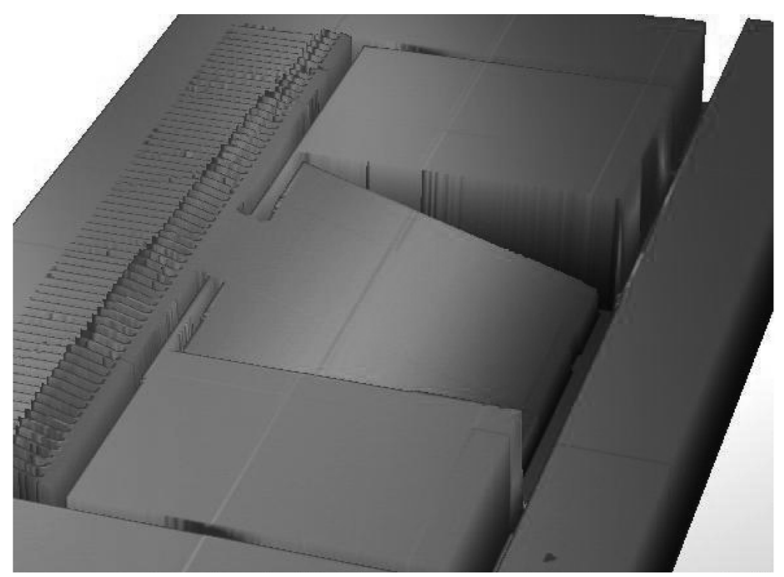

(a)

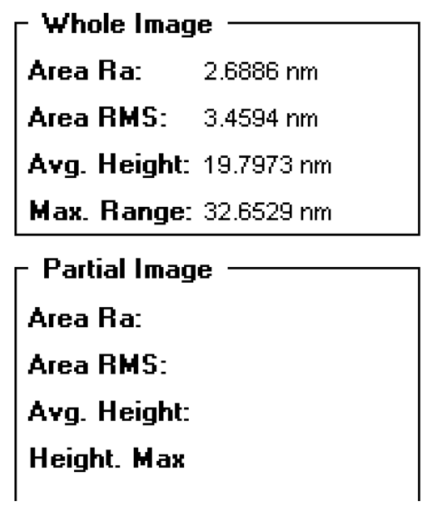

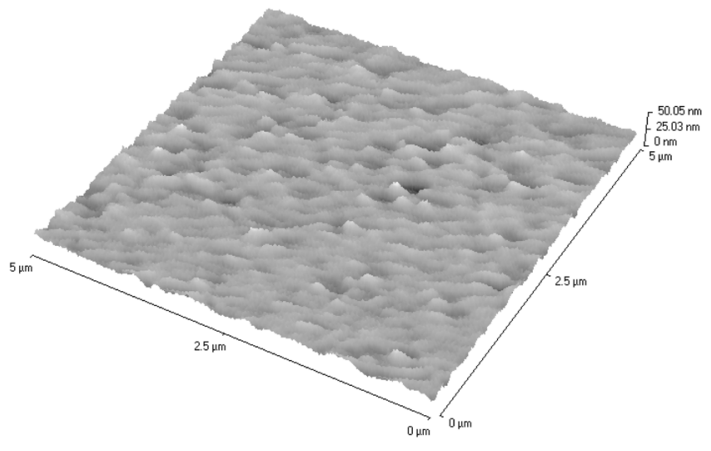

(b)

Fig. 12. Structure and surface inspection using white light interferometer and AFM. (a) 3-D image generated from WYKO measurement. (b) The surface roughness measurements of mirror surface on the presumptive contact positions of the pillar using AFM. The scanned area is $5 \times 5 \mu \mathrm{m}^{2}$ and the average surface roughness is $2.7 \mathrm{~nm}$.

dimensions on the 50- $\mu$ m-thick single-crystal-silicon (100) SOI device layer. An SEM photograph of this chip is shown in Fig. 13. The dimensions of all the test mirrors were 50 $\mu \mathrm{m} \times 600 \mu \mathrm{m}$ times $1200 \mu \mathrm{m}$, and each mirror was supported by two identical torsion bars attached symmetrically on the longer sides. The dimensions of torsion bars were picked from stress calculation such that the test structure arrays include different torsion bar groups with the elastically induced stresses slightly smaller than $\sigma_{\mathrm{m}}$, slightly larger than $\sigma_{\mathrm{m}}$ and well above $\sigma_{\mathrm{m}}$. After loading with the processing chips, the sandwiched chips were heated above room temperature using the same annealing schedule used for device chips and described earlier.

The test results in Fig. 14 show that elastic recovery depends both on the torsion bar dimension and the amount of initial elastic deformation. Each plotted data point is an averaged value of eight measurements of identical test structures from four different batches of plastic deformation processes. The applied elastic deformation is calculated from the pillar height and location, and the plastically deformed angle was measured using white light interferometer. In the first plot (a), the location of $45 \mu \mathrm{m}$-height pillar to give elastic strain is varied from $100 \mu \mathrm{m}$ to $350 \mu \mathrm{m}$ from the edge of the mirrors for the same dimension of $16 \mu \mathrm{m} \times 50 \mu \mathrm{m} \times 1000 \mu \mathrm{m}$ torsion bars. As depicted in Fig. 11, the farther position of pillar from the edge gives larger elastic deformation on the torsion bars. The scale on the

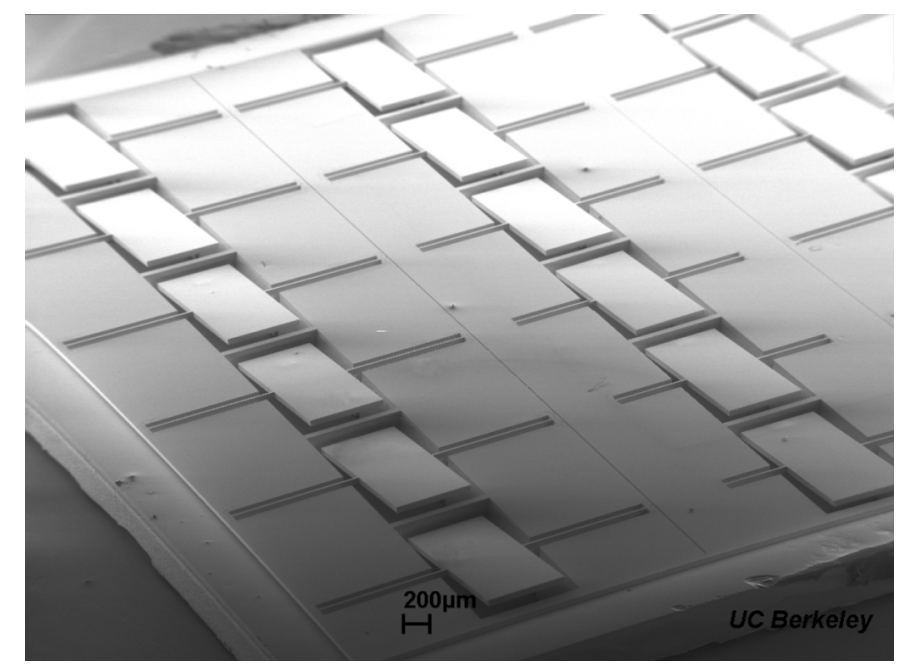

Fig. 13. Test structures used to characterize springback; the first and second of three arrays contain varied width and length of beams with identical angle deformed elastically. For the third array, varied elastically deformed angle is applied on each mirror to induce different stresses in torsion bars.

left-hand side of the $y$ axis shows the rotation angles of the mirrors while that on the right hand side shows the percentages of the amount of springback with respect to the applied elastic tilt angles. For an elastic tilt angle smaller than $10^{\circ}$, larger strain 
Pillar position and Springback

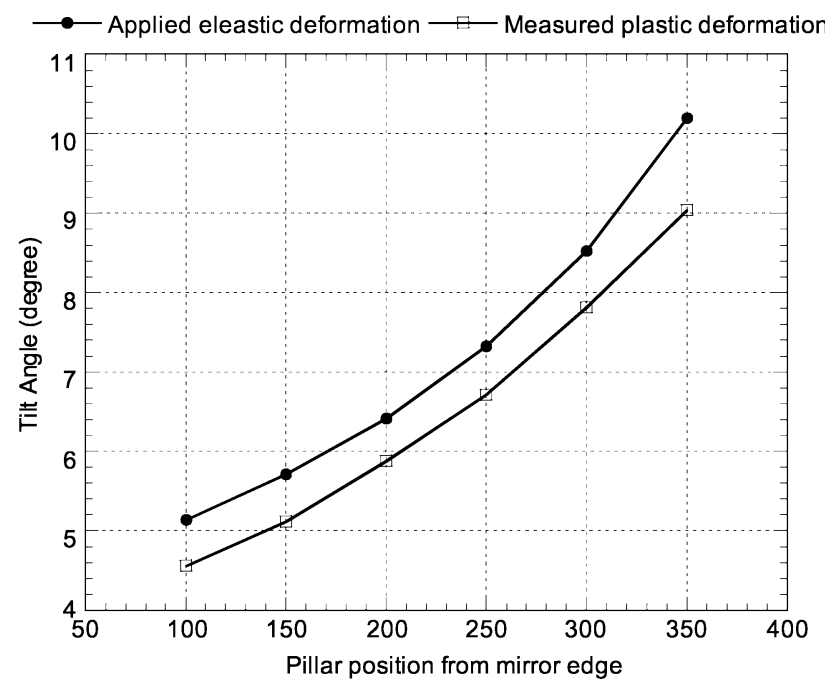

(a)

Beam length and Springback

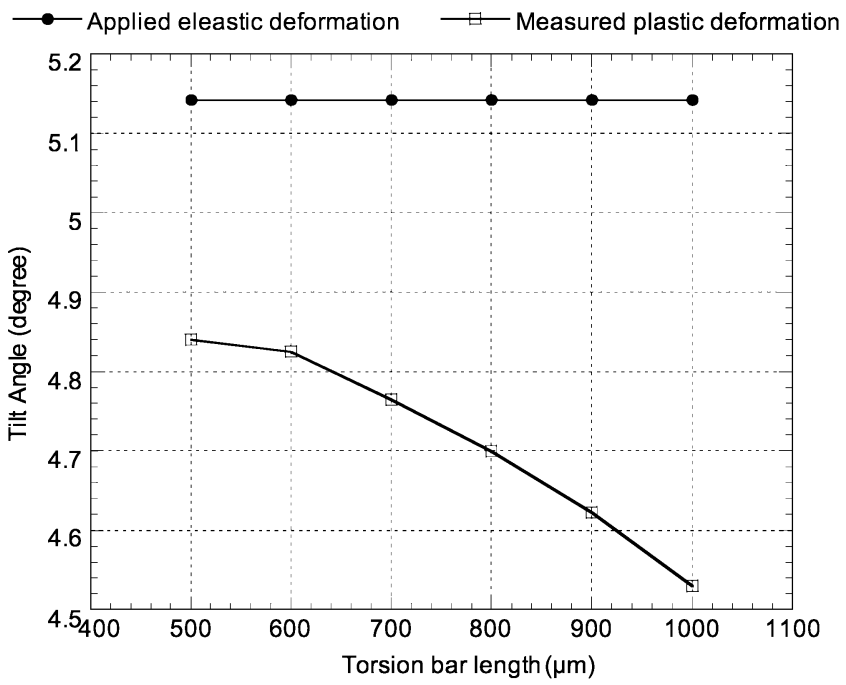

(c)

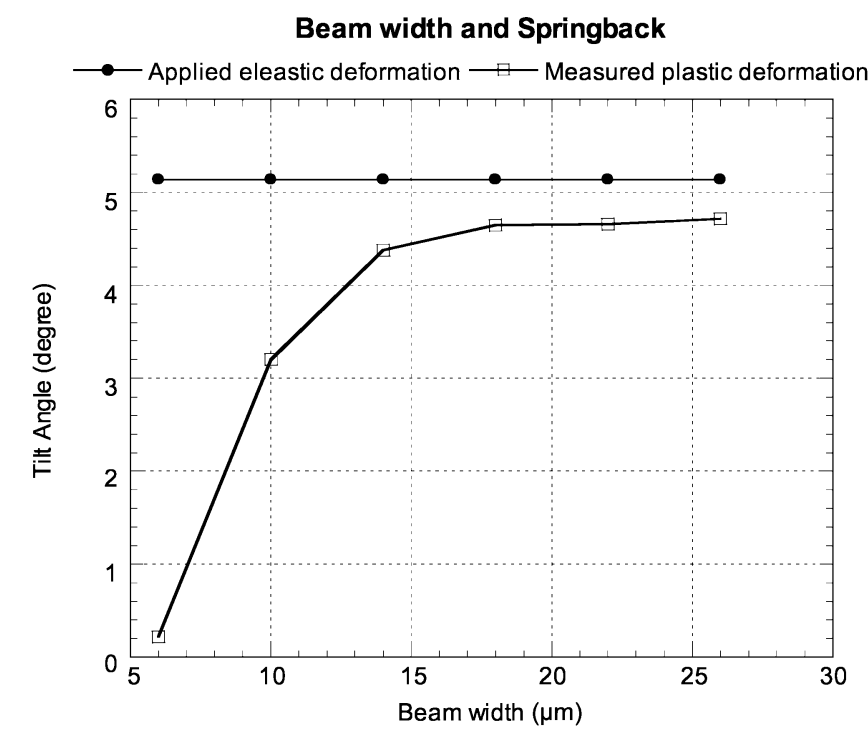

(b)

Shear stress VS \% springback

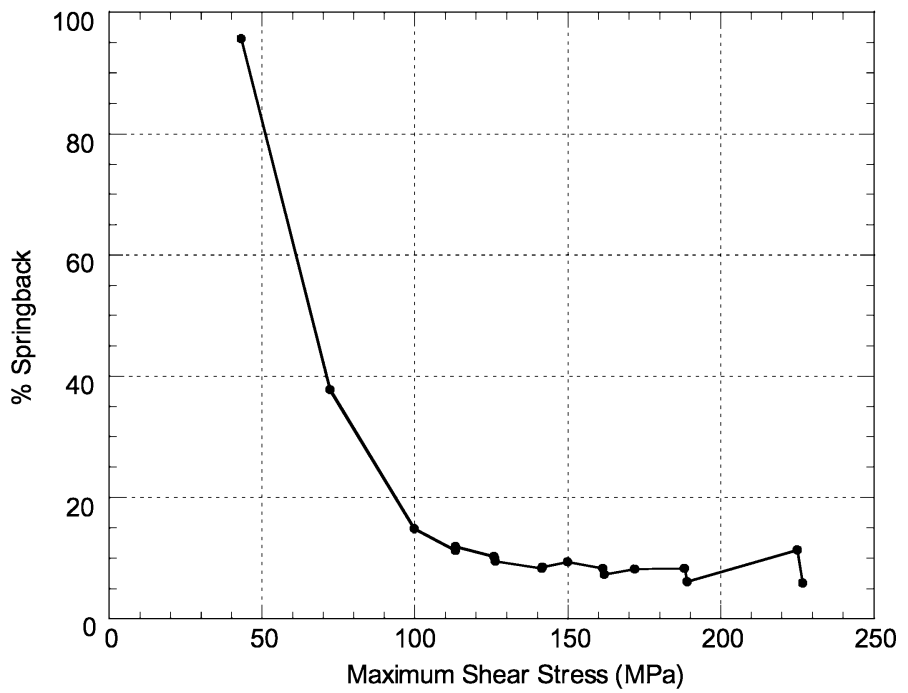

(d)

Fig. 14. Applied initial deformation angle set by the pillar and measured plastically deformed angle depending on the torsion bar dimension. The set angle is calculated from the pillar height and the location of the pillar, and the plastically deformed angle is measured using white light interferometer. (a) Location of pillar to give elastic strain is varied from 100 to $350 \mu \mathrm{m}$ from the edge of the mirrors. (b) Width of torsion bars is varied from 6 to $26 \mu \mathrm{m}$ for the same length of $1000 \mu \mathrm{m}$ and elastically deformed tilt angle applied. (c) Lengths of the torsion bars are varied from 500 to $1000 \mu \mathrm{m}$ for the same width of $16 \mu \mathrm{m}$ and elastically deformed tilt angle applied. (d) Springback percentage values with respect to the initial deformation angle set by the pillar versus the shear stress induced in torsion bars is plotted based on the measurements in (a), (b), and (c). The stress values are calculated from the set angle and torsion bar dimensions.

gives smaller springback/strain ratio. However, for the test structures with larger than $10^{\circ}$ of elastic torsion, we have found that elastic recovery saturates or even increases slightly. This means the flow stress $\sigma_{\mathrm{f}}$ in Fig. 3 in fact increases for a larger shear strain from torsion, and other researchers observed similar test results for a tensile test [11] and a bending test [16] of single crystalline silicon. Similarly, Fig. 14(b) is plotted with respect to the width change of torsion bars from $6 \mu \mathrm{m}$ to $26 \mu \mathrm{m}$ for the same length of $1000 \mu \mathrm{m}$ and elastically deformed tilt angle applied. The elastic recovery for $6 \mu \mathrm{m}$ bar is larger than $95 \%$ of applied elastic torsion since the largest stress induced on this thin bar is just as large as the yield stress at high temperature.
As the width increases, the amount of elastic recovery decreases and approaches to about $8 \%$ of given elastic deformation since the maximum shear stress in the torsion bar also increases. We also plotted applied elastic deformation, plastic deformation, and percentages of springback against different lengths of the bars in Fig. 14(c). Here the lengths of the torsion bars (width of $16 \mu \mathrm{m}$ ) vary from 500 to $1000 \mu \mathrm{m}$, and as we expected, the amount of springback is larger for longer bars on which smaller stresses are induced.

To extend the usage of the measurement data to general cases, springback percentages are plotted in Fig. 14(d) in terms of maximum shear stresses induced in the torsion bars from the 


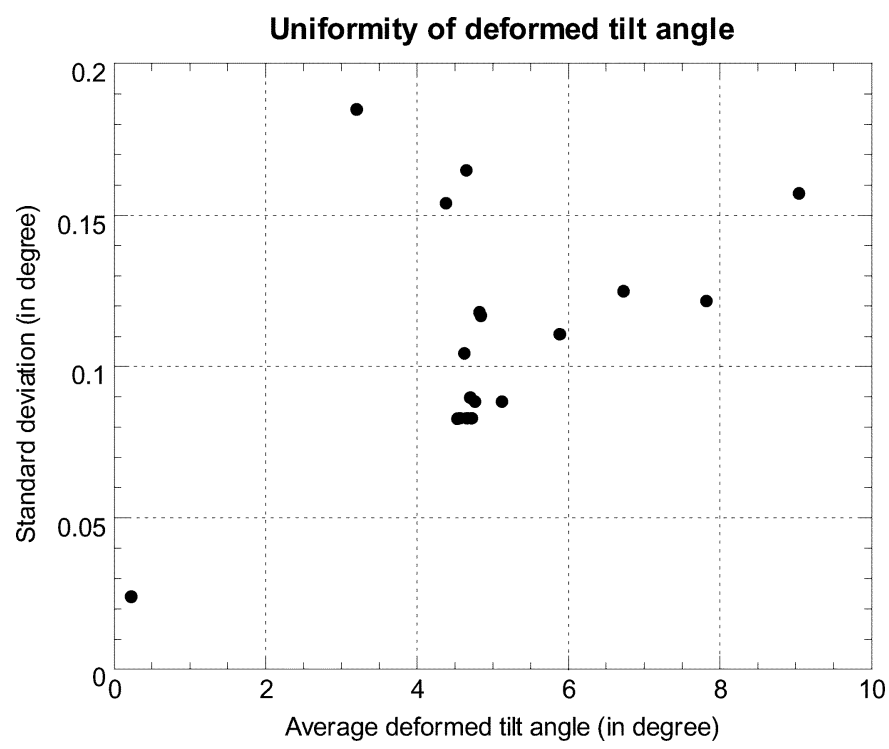

Fig. 15. Standard deviation of plastically formed tilting angles for the tested mirrors in Fig. 14. The angles are measured using white light interferometer with the vertical resolution of sub nanometer.

applied elastic deformation. The exact form of the torsion bar stiffness and stress with uniform rectangular cross section are represented as a summation of infinite series of terms. However, good approximation of the torsional stiffness and maximum shear stress of a torsion bar can be written as [17]

$$
\begin{aligned}
M= & k_{\theta} \theta=\frac{a b^{3} G}{L}\left[\frac{16}{3}-3.36 \frac{b}{a}\left(1-\frac{b^{4}}{12 a^{4}}\right)\right] \theta \\
\tau= & \frac{3 M}{8 a b^{2}}\left[1+0.6095 \frac{b}{a}+0.8865\left(\frac{b}{a}\right)^{2}\right. \\
& \left.-1.8023\left(\frac{b}{a}\right)^{3}+0.9100\left(\frac{b}{a}\right)^{4}\right]
\end{aligned}
$$

where $\mathrm{G}=$ shear modulus, $\mathrm{q}=$ angle of rotation, $2 \mathrm{a}=$ length of the long side, $2 \mathrm{~b}=$ length of the short side. Fig. 14(d) is generated from the data plotted in (a)-(c) using (1) and (2). Again, it is obvious that the springback effect is significant at lower stress level and converges to a finite value. These figures provide design guidelines for the plastically deformed AVC actuators, especially for achieving specific plastically deformed tilt angles.

Standard deviations of the plastically deformed tilt angles with respect to the average values are plotted in Fig. 15. The angles were measured on the structures used for the plots in Fig. 13. The typical standard deviation is in the range of $0.08^{\circ}-0.19^{\circ}$. There seems to be no correlation between the tilt angle and the uniformity. Possible sources of deviation are: particles entrapped between the device substrate and lid substrate, or the tolerance for self-alignment between the depressions on the device substrate and the protrusions on the lid substrate. The maximum tolerance used in our process is $\pm 4 \mu \mathrm{m}$, and the uniformity can be improved by mating the lid and device substrates with more tight tolerances and in a cleaner environment.

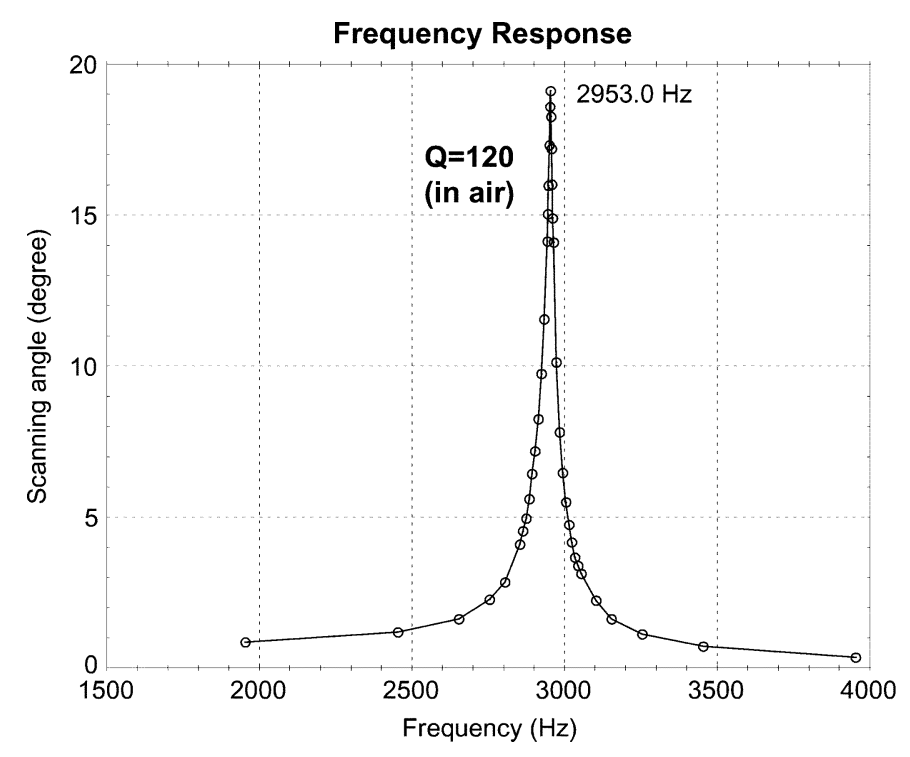

Fig. 16. Frequency response of the type II microactuator.

\section{EXPERIMENTAL RESULTS AND RELIABILITY}

The measured frequency response of the type II actuator with the initial tilt angle of $5.22^{\circ}$ is presented in Fig. 16. The mirror, whose dimensions were $1000 \mu \mathrm{m} \times 1000 \mu \mathrm{m}$, was driven with $40 \mathrm{~V}_{\mathrm{dc}}$ and $13 \mathrm{~V}_{\mathrm{pp}}$. The maximum scanning angle of $19.2^{\circ}$ is achieved at the resonant frequency of $2953 \mathrm{~Hz}$. The quality factor measured in air is 120 . For the type I actuator with the initial tilt angle of $3.75^{\circ}, 24.8^{\circ}$ of optical scanning angle was measured at the resonant frequency of $2499 \mathrm{~Hz}$ and with the 45 $\mathrm{V}_{\mathrm{dc}}$ and $20 \mathrm{~V}_{\mathrm{pp}}$ driving voltage. For both designs, the moving and fixed combs were $50 \mu \mathrm{m}$ thick.

The plastically deformed angular vertical comb actuator has proven to operate very reliably and stably. Its design is simple, and its fabrication is straightforward. To test long-term reliability, we resonated type II scanner at full amplitude for 5.1 billion cycles, using the same driving voltages used to measure the frequency response in Fig. 16. For this test, the mirror actuator was set to be operated at its resonance frequency with a 1-mW helium-neon laser beam directing at the mirror surface and reflected on a distal screen $135 \mathrm{~cm}$ away from the actuator to measure the scanning angle constancy over the long period of time. In Fig. 17 the changes in resonant frequency and scanning angle were measured at every 255 million cycles that corresponds to $24 \mathrm{~h}$ of operation. The maximum variations of resonant frequency and scanning angle were $0.064 \%$ and $3.6 \%$, respectively. The error bars on the frequency curve are shown because the change of the amplitude of the reflected laser pattern on the screen was undetectable to $0.2 \mathrm{~Hz}$ variation of the operational frequency.

This experiment was performed with a mirror actuator unpackaged and in the lab. It is not obvious if this variation is from the degradation of the device or from other possible effects such as temperature variation, particle-contaminations or variation in humidity. However, considering that the amount of angle and frequency variation is very small and the actuator was not packaged, the scanning mirror actuators made by plastic deformation method are robust and reliable. 


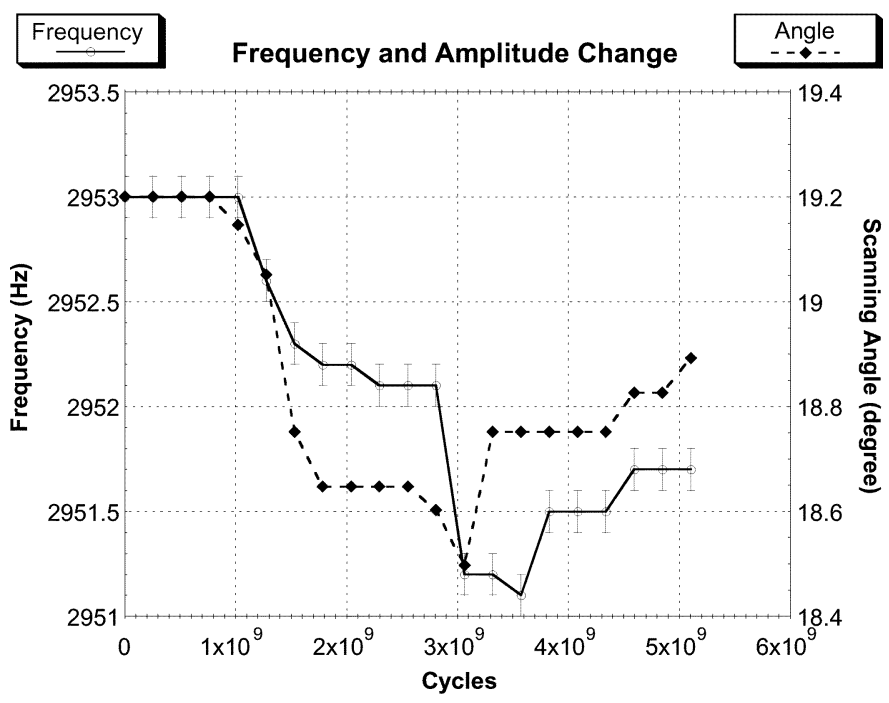

Fig. 17. Measured scanning angle and resonant frequency of the microactuator over 5 billion cycles; the gap between each data point is 255 million cycles ( $1 \mathrm{~d}$ ).

\section{CONCLUSION}

We have presented a straightforward batch process to fabricate angular vertical comb-drive actuators in single-crystal silicon using SOI chips. A separately processed chip is used to apply torque to the torsion bars which are plastically deformed at an anneal temperature of $900{ }^{\circ} \mathrm{C}$. We have studied actuators formed by this method using scanning mirrors. The fabrication and characterization of angular vertical combs for scanning-mirror applications have been accomplished and the measured dynamic performance of the actuator was comparable to or superior to that reported in earlier vertical-comb actuators. Elastic recovery of the torsion bars that follows plastic deformation process was quantitatively characterized for a variety of torsion bar dimensions. We believe this can be guidelines for the design of plastically deformed AVC actuators, especially for achieving specific plastically deformed tilt angles.

A reliability test on an unpackaged mirror actuated through more than five billion cycles of operation showed less than $0.064 \%$ decrease in the resonant frequency. The actuators have many other potential applications where vertically driven linear or torsional motions are required as well as the MEMS scanning mirrors presented in this paper.

\section{REFERENCES}

[1] R. A. Conant, P. M. Hagelin, U. Krishnamoorthy, M. Hart, O. Solgaard, K. Y. Lau, and R. S. Muller, "A raster-scanning full-motion video display using polysilicon micromachined mirrors," Sens. Actuators, vol. A83, no. 1-3, pp. 291-296.

[2] V. Milanovic, M. Last, and K. S. J. Pister, Torsional Micromirrors with Lateral Actuators, Munich, Germany, pp. 1290-1301. Transducers 01.

[3] D. Hah, S. Huang, H. Nguyen, H. Chang, J.-C. Tsai, M. C. Wu, and H. Toshiyoshi, "Low voltage MEMS analog micromirror arrays with hidden vertical comb-drive actuators," in 2002 Solid-State Sens., Actuator and Microsystems Workshop, pp. 11-14.

[4] J. A. Yeh, H. Jiang, and N. C. Tien, "Integrated polysilicon and DRIE bulk silicon micromachining for an electrostatic torsional actuator," $J$. Microelectromech. Syst., vol. 8, no. 4, pp. 456-465, Dec. 1999.

[5] R. A. Conant, J. T. Nee, K. Lau, and R. S. Mueller, "Flat high-frequency scanning micromirror," in 2000 Solid-State Sensor and Actuator Workshop, Hilton Head, SC, pp. 6-9.
[6] U. Krisnamoorthy and O. Solgaard, "Self-aligned vertical comdrive actuators for optical scanning micromirrors," in 2001 Int. Conf. Opt. $M E M S$, p. 41.

[7] H. Xie, Y. Pan, and G. K. Fedder, "A CMOS-MEMS micromirror with large out-of-plane actuation," in 2001 ASME Int. Mech. Eng. Congr. Expo., vol. 3, pp. 89-92.

[8] P. R. Patterson, D. Hah, H. Nguyen, H. Toshiyoshi, R.-M. Chao, and M. C. Wu, "A scanning micromirror with angular comb drive actuation," Tech. Dig. MEMS 2002 IEEE Int. Conf., pp. 544-547, 2002.

[9] K. Isamoto, T. Makino, K. Kato, A. Morosawa, C. Chong, H. Fujita, and H. Toshiyoshi, "A two-mask process for self-assembled vertical comb drive mirrors," in IEEE/LEOS Int. Conf. Opt. MEMS and Their Applicat., 2004, pp. 172-173.

[10] J. Kim, D. Christensen, and L. Lin, "Selective stiction based vertical comb actuators," in Tech. Dig. MEMS 2005 IEEE Int. Conf. Micro Electro Mech. Syst., 2005, pp. 403-406.

[11] J. R. Patel and A. R. Chaudhuri, "Macroscopic plastic properties of dislocation-free germanium and other semiconductor crystals," J. Appl. Phys., vol. 34, no. 9, pp. 2788-2799, 1963.

[12] M. M. Farooqui and A. G. R. Evans, "Polysilicon microstructures," in Proc. IEEE Micro Electro Mech. Syst., 1991, pp. 187-191.

[13] M. A. Huff, A. D. Nikolich, and M. A. Schmidt, "A threshold pressure switch utilizing plastic deformation of silicon," in Transducers'91 Int Conf. Solid-State Sens. Actuators, 1991, pp. 177-180.

[14] Y. Fukuta, D. Collard, T. Akiyama, E. H. Yang, and H. Fujita, "Microactuated self-assembling of 3D polysilicon structures with reshaping technology," in Proc. IEEE. 10th Ann. Int. Workshop on Micro Electro Mech. Syst., 1997, pp. 477-481.

[15] E. Gartner, J. Fruhauf, B. Hannemann, and E. Jansch, "Mounting of Si-chips with plastically bent cantilevers," in Transducers'01 Eurosensors XV. 11th Int. Conf. Solid-State Sens. Actuators, vol. 1, 2001, pp. 206-209.

[16] J. Fruhauf, E. Gartner, and E. Jansch, "New aspects of the plastic deformation of silicon-prerequisites for the reshaping of silicon microelements," Appl. Phys. A (Mater. Sci. Process.), vol. A68, no. 6, pp. 673-679, 1999.

[17] W. C. Young and R. G. Budinas, Roark's Formulas for Stress and Strain. New York: McGraw-Hill, 2002.

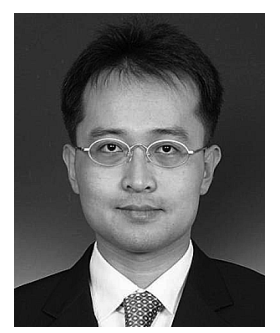

Jongbaeg Kim received the B.S. degree in mechanical engineering from Yonsei University, Seoul, Korea, in 1997, the M.S. degree in mechanical engineering from the University of Texas, Austin, TX, in 1999, and the Ph.D. degree in mechanical engineering from the University of California, Berkeley, in 2004.

He was with DiCon Fiberoptics, Inc., Richmond, CA, from 2004 to 2005, where he designed and developed high-performance microactuators for telecommunication applications. He then joined the Yonsei University, where he is currently an Assistant Professor with the School of Mechanical Engineering. His research interests are mechatronic system dynamics, modeling, design and fabrication of MEMS, and nanotechnology.

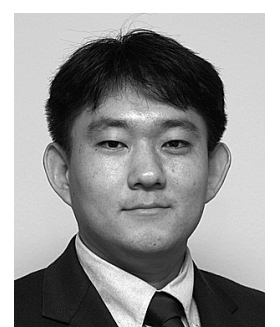

Hyuck Choo received the B.S. and M. Eng. degrees in 1996 and 1997, respectively, in electrical engineering from Cornell University, Ithaca, NY.

$\mathrm{He}$ is currently pursuing the Ph.D. degree in electrical engineering and computer sciences at the University of California, Berkeley (UC Berkeley), under Prof. R. S. Muller's supervision. Before joining UC Berkeley, he was with Kionix, Inc., Ithaca, NY, as a MEMS test engineer. His current research interests are microlens and microscanner systems and their applications to ocular refractive surgeries, biomedical imaging systems, high-definition displays, and next-generation wavefront sensors. He has two nonprovisional U.S. and international patents (in application) on his microlens and microscanner systems. He

Mr. Choo won the UC Berkeley EECS Lim Prize in 2001 (Best Performance on the Pre-Doctoral Exam) and is the two-time winner of the Berkeley Sensor and Actuator Center presentation award. 


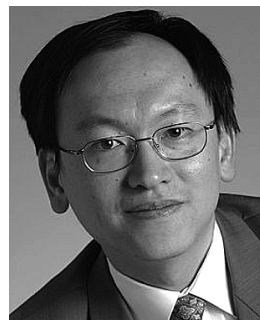

Liwei Lin (S'92-M'93) received the B.S. degree from National Tsing-Hua University, Taiwan, in 1986, and the M.S. and Ph.D. degrees in mechanical engineering from the University of California, Berkeley (UC Berkeley), in 1991 and 1993, respectively.

He was with BEI Electronics Inc. USA from 1993 to 1994 working in microsensors research and development. He was an Associate Professor with the Institute of Applied Mechanics, National Taiwan University, Taiwan, from 1994 to 1996, and an Assistant Professor with the Mechanical Engineering Department, University of Michigan, from 1996 to 1999 . He joined UC Berkeley in 1999 and is now a Professor with the Mechanical Engineering Department and is Co-Director of the Berkeley Sensor and Actuator Center, an NSF/Industry/University Research Cooperative Center. His research interests are in design, modeling, and fabrication of micro/nano structures, micro/nano sensors, and micro/nano actuators, as well as mechanical issues in micro/nano electromechanical systems. He holds eight U.S. patents.

Dr. Lin is the recipient of the 1998 NSF CAREER Award for research in MEMS Packaging and the 1999 ASME Journal of Heat Transfer Best Paper Award for his work on micro scale bubble formation. He is a Subject Editor for the IEEE/ASME JouRNAL OF MicROELECTROMECHANICAL SYSTEMS and the North and South America Editor for Sensors and Actuators-A Physical. He was the founding chairman of the ASME MEMS division in 2004 and is a Fellow of the American Society of Mechanical Engineers (ASME).

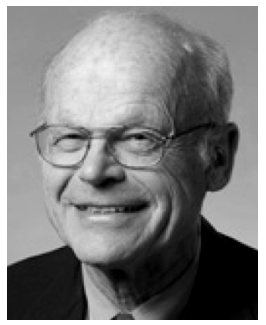

Richard S. Muller (S'57-M'58-SM'70-F'88LF'97) received the mechanical engineer's degree from Stevens Institute of Technology, Hoboken, NJ, the M.S. (electrical engineering) and Ph.D. (electrical engineering and physics) degrees in 1962 from the California Institute of Technology, Pasadena.

He was a Member of the Technical Staff at Hughes Aircraft Company before joining the faculty at the University of California, Berkeley, where he concentrated his research on the physics of integrated-circuit devices. He coauthored, with Dr. T. I. Kamins of the Hewlett-Packard Company, Device Electronics for Integrated Circuits. A third edition of this book (which has been translated into five languages) was published in 2003. In the late 1970s, he began research in the area now known as MEMS and, together with Professor R. M. White, he founded the Berkeley Sensor and Actuator Center in 1986. His present research focus is on optical MEMS.

Dr. Muller wrote the proposal to establish the IEEE/ASME JOURNAL OF MicroelectromeCHANICAL SySTEMS (JMEMS) and is the current Editor-in-Chief. A member of the U.S. National Academy of Engineering, he received a career MEMS Award at Transducers'97, as well as the IEEE Brunetti Award (1998 with R. T. Howe), NATO and Fulbright Professorships, and a von Humboldt Research Award at TU Berlin in 1994. Other awards include the Berkeley Citation and the Renaissance Award from Stevens Institute of Technology, where he served as Trustee 1996-2005. He has been a member of the National Materials Advisory Board and served on several National Research Council study panels, as well as chairing a 1997 panel for which he acted as editor of a widely distributed report on the promises and challenges of MEMS. He is a Member of the American Society of Mechanical Engineers (ASME). 